Journal of Advanced Research in Fluid Mechanics and Thermal Sciences

Journal homepage: www.akademiabaru.com/arfmts.html ISSN: $2289-7879$

\title{
Peristaltic Motion of Eyring-Powell Nano Fluid with Couple Stresses and Heat and Mass Transfer Through a Porous Media Under the Effect of Magnetic Field Inside Asymmetric Vertical Channel
}

\author{
Nabil T. Eldabe ${ }^{1}$, Kawther A. Kamel ${ }^{2}$, Shaimaa F. Ramadan², Rabab Ahmed Saad²,* \\ Department of Mathematics, Faculty of Education, Ain Shams University, Heliopolis, Cairo, Egypt \\ Department of Mathematics, Faculty of Science (Girls), Al-Azhar University, Nasr-City, Cairo, Egypt
}

\section{ARTICLE INFO}

\section{Article history:}

Received 19 November 2019

Received in revised form 11 January 2020

Accepted 12 January 2020

Available online 30 March 2020

\section{Keywords:}

Peristaltic flow; heat absorption; couple stress fluid; porous medium; nanofluid; concentration; Reynolds number

\section{ABSTRACT}

\begin{abstract}
A Numerical study of peristaltic transport of heat and mass transfer for a couple stresses non-Newtonian nano fluid through porous media inside asymmetric channel was investigated. The system is stressed by an external uniform magnetic field. This model is solved numerically subjected to an approbate boundary conditions using a Rung-Kutta-Merson method under assumption of the long wave length and low range of Reynolds number. The velocity, temperature and concentration of nanoparticles are obtained as a function of the physical parameters of the problem. The effects of these parameters on these solutions are discussed numerically and illustrated graphically through some graphs It is clear that the effects of the physical parameters of the problem on these solutions play an important rule to control the obtained solutions. The velocity profile decreases when Hartmann number increasing. This seems realistic, because the magnetic field acts in the transverse direction to the flow and magnetic force resists the flow, The velocity increasing when the fluid material parameter increasing because fluid becomes less viscous, The variation of temperature increasing with increasing values of Brownain motion parameter and porosity parameter, The variation of Concentration decreasing with increasing of thermophoresis parameter and Radiation parameter inside the used channel.
\end{abstract}

Copyright @ 2020 PENERBIT AKADEMIA BARU - All rights reserved

\section{Introduction}

Nanotechnology is a huge scientific revolution no less than the industrial revolution that moved man to the era of machinery or the revolution of technology that moved man to the age of space, communications and the Internet, and a comprehensive development in various fields and all

\footnotetext{
* Corresponding author.

E-mail address: rabab1993ahmed@gmail.com (Rabab Ahmed)
} 
branches of science. At a lower cost and higher quality, this ability will be the key to scientific advancement that will transform life in such a way that one cannot imagine all its dimensions today. In medicine, gold-plated nanostructures are used to destroy cancer cells. The length of these nanostructures is about 120 nanometers, which is 170 times smaller than the size of a cancer cell. When injected into the body, these nanostructures are automatically bound to cancer cells, and are then exposed to radiation. Infrared laser, which works to heat and raise the temperature of gold, which leads to the combustion and death of these cells, and this method, is characterized by accuracy and positional because of the small Nano cellular envelopes for the cells and the concentration of diseased cells only, which makes healthy cells away from the risks of effects Lateral. Kothandapani and Prakash [1] have studied the nanofluids particles on the peristaltic flow of Williamson fluid with thermal radiation. Nield and Kuznetsov [2] investigated the natural convective boundary layer flow of nanofluid over a flat vertical plate. The effects of endoscope on the peristaltic transport of nanofluids have been studied by Akbar and Nadeem [3]. Gireesha et al., [4] have studied the effects of dust particles suspended in a nano fluid flow past a stretching surface. Noreen sher Akbar et al., [5] studied peristaltic flow of a nano fluid in anon-uniform tube. Nadeem et al., [6] studied effects of heat and mass transfer on per is taltic flow of a nano fluid between eccentric cylinders.

Peristaltic motion is a wave of contractions and diastoles that push the fluid, making it easier to move along the path. Peristalsis is an intrinsic property of various biological systems with smooth muscles as well as tubes that transport fluid through the vessels as a result of the motive movement. A clear example of this movement is the movement of urine from the kidney to the bladder, the movement of food within the gastrointestinal tract, and also the movement of eggs within the fallopian tube and so on. Hayat et al., [7] have studied peristaltic flow of couple-stress fluid with heat and Mass Transfer: An application in biomedicine. Noreen [8] discussed the Peristaltic flow of a tangent hyperbolic fluid with convective boundary condition. Nadeem et al., [9] have studied peristaltic flow of hyperbolic tangent fluid in a diverging tube with heat and mass transfer. Eldabe and Abou-zeid [10] have studied magneto hydrodynamic peristaltic flow with heat and mass transfer of micro polar biviscosity fluid through a porous medium between two co-axial tubes. Eldabe and Abou-zeid [11] have studied the radially varying magnetic field effect on peristaltic motion with heat and mass transfer of a non-Newtonian fluid between two Co-axial tubes. These are attracted several investigators to studied the peristaltic transport under long wavelength and low Reynolds number approximations.

The analysis of the fluid flow in porous media has evolved throughout the years along two fronts: experimental and analytical. Physicists, engineers and hydrologists have experimentally examined the behavior of various fluids as they flow through porous media ranging from sand packs to fused Pyrex glass. On the basis of their analyses they have attempted to formulate laws and correlations that can then be utilized to make analytical predictions for similar systems. Eldabe et al., [12] have investigated numerical study of viscous dissipation effect on free convection heat and mass transfer of MHD non-Newtonian fluid flow through a porous medium. Eldabe et al., [13] have studied effects of chemical reaction on a peristaltic motion of MHD biviscosity fluid with heat and mass transfer through a porous medium in an inclined asymmetric Channel. Abou-zeid [14] discussed the magneto hydrodynamic boundary layer heat transfer to a stretching sheet including viscous dissipation and internal heat generation in a porous Medium. Eldabe et al., [15] have studied The Hall effects on the peristaltic transport of williamson fluid through a porous medium with heat and mass transfer. Ramesh and Devakar [16] studied the magneto hydrodynamic peristaltic transport of couple stress fluid through porous medium in an inclined asymmetric channel with heat transfer.

The mathematical forms of the relationships designed to describe flow behavior of the fluids. will vary depending upon characteristics of the fluid. Hina et al., [17] have studied the peristaltic transport 
of Powell-Eyring fluid in a curved channel with heat/mass transfer and wall properties. The EyringPowell model [18] although more mathematically complex, has certain advantages over the second grade, Maxwell, Power-law and Micropolar fluid models. Eyring-Powell model is derived from the kinetic theory of liquids rather than the empirical relations. It correctly reduces to Newtonian behavior for low and high shear stress. Haider et al., [19] have studied the Unsteady incompressible Couette flow problem for the Eyring-Powell model with porous walls. Eldabe et al., [20] have studied the peristaltic transport of magnetohydrodynamic Carreau nanofluid with heat and mass transfer inside asymmetric channel. Siva et al., [21] have studied the combined effect of heat and mass transfer on MHD peristaltic transport of a couple stress fluid in an inclined asymmetric channel through a porous medium. Eldabe et al., [22] have studied the hall effect on peristaltic flow of third order fluid in a porous medium with heat and mass transfer. Alharbi et al., [25] have studied the entropy generation in MHD Eyring-Powell fluid flow over an unsteady oscillatory porous stretching surface under the impact of thermal radiation and heat source/sink. The hydromagnetic flow and heat transfer of Eyring-Powell fluid over an oscillatory stretching sheet with thermal radiation is investigated by Khan and Ali [26].

The main aim of this work is to study the peristaltic flow of Eyring-Powell nano fluid with heat and mass transfer through porous medium in asymmetric vertical channel. The couple stresses, radiation, uniform magnetic field, porous medium is taken in consideration. The analysis is performed under the long wavelength and low Reynolds number approximations. Then the numerical techniques based on the Rung-Kutta-Merson method is used to obtain the solution of the governed equations.

\section{Applications}

Nanotechnology plays a major role in improving the geometry of living tissues and cell therapy, including the use of living cells or natural or synthetic compounds that are grown within the body of the organism. Some researchers are now experimenting with experiments using silicon nanoparticles to stop the body's immune system from recognizing foreign cells. These capsules block antibodies from the body's immune system while enough insulin is released by nanoparticles into the blood. There has been a lot of researches on the importance of nanotechnology in diagnosing cancer cells [23] as shown in Figure 1. Nanoparticles also contribute to stronger, lighter, cleaner and smarter surfaces and systems. They are already used in the manufacture of crack-resistant paints, transparent sunscreens, stain-resistant fabrics and self-cleaning windows. 


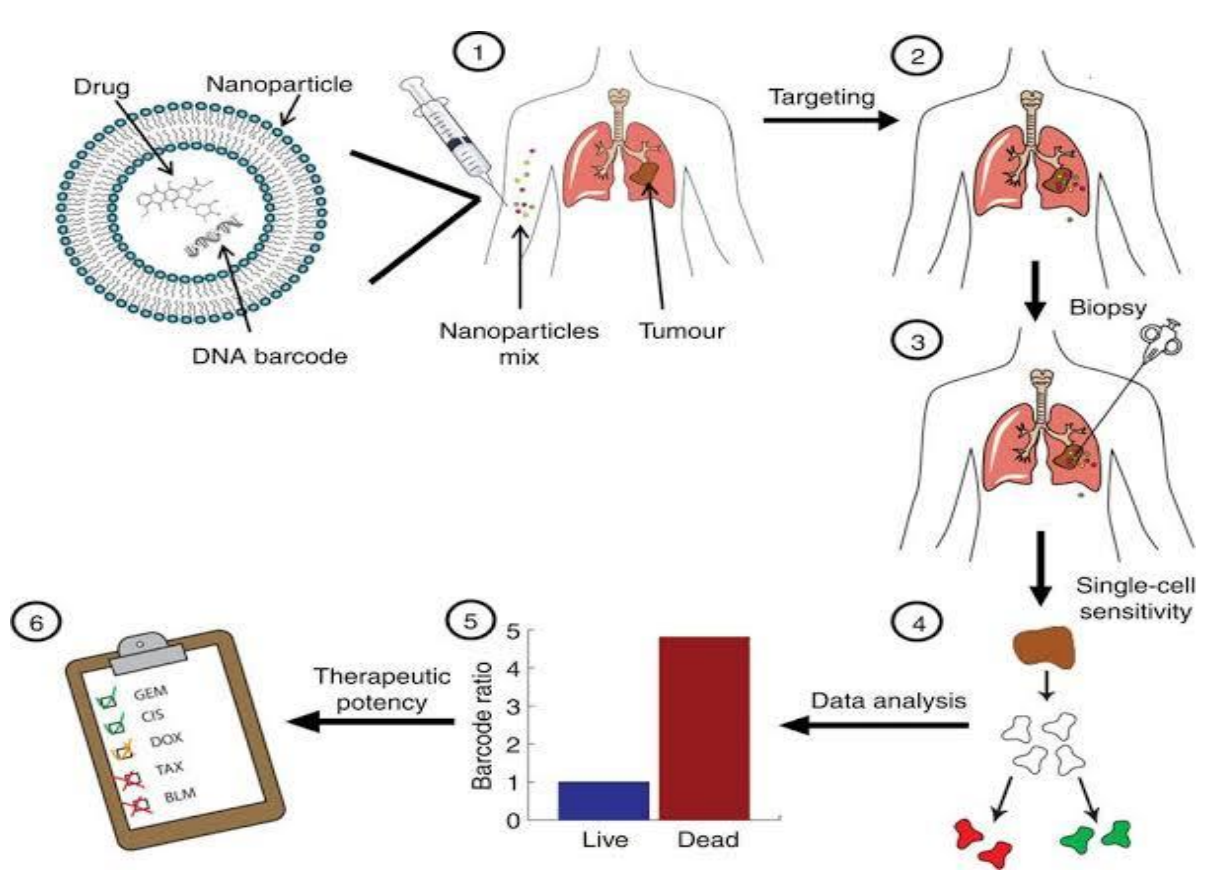

Fig. 1. Using nanotechnology to probe the sensitivity of cancer to medicines

Peristalsis, involuntary movements of the longitudinal and circular muscles, primarily in the digestive tract but occasionally in other hollow tubes of the body, that occur in progressive wavelike contractions. Peristaltic waves occur in the oesophagus, stomach, and intestines. The waves can be short, local reflexes or long, continuous contractions that travel the whole length of the organ, depending upon their location and what initiates their action. In the oesophagus, peristaltic waves begin at the upper portion of the tube and travel the whole length, pushing food ahead of the wave into the stomach. Particles of food left behind in the oesophagus initiate secondary peristaltic waves that remove leftover substances [24] as shown in Figure 2. One wave travels the full length of the tube in about nine seconds. The peristaltic-wave contractions in the oesophagus of humans are weak compared with those of most other mammals. In cud-chewing animals, such as cows, reverse peristalsis can occur so that the food is brought back from the restore it.

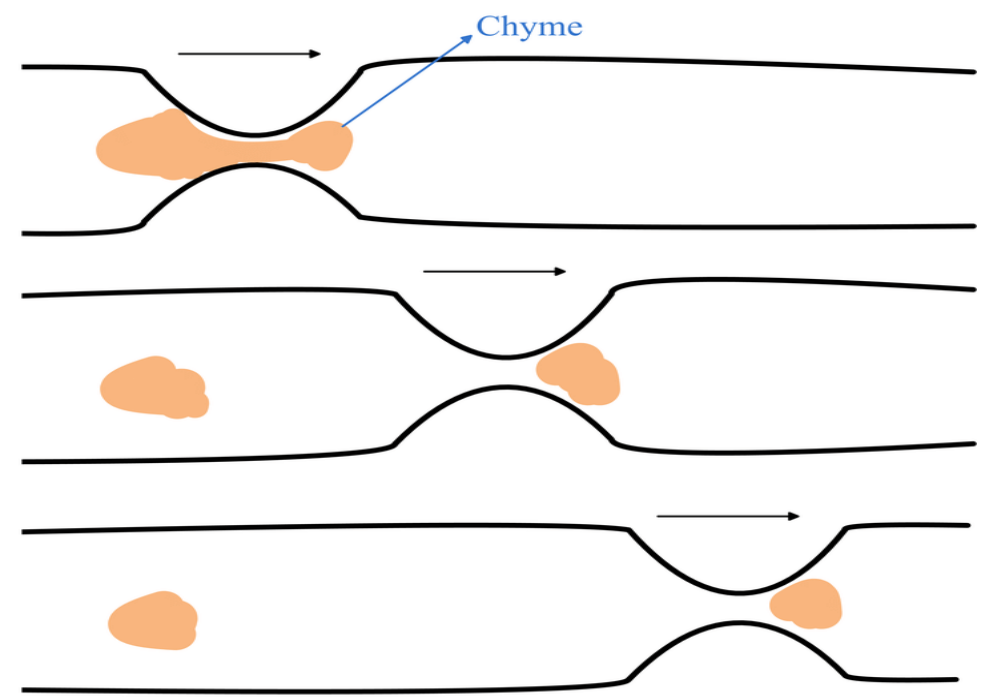

Fig. 2. Peristalsis motion of GI tract 


\section{Mathematical Formulation of the Problem}

The Cartesian coordinates represented in $\mathrm{X}$ and $\mathrm{Y}$ are considered as shown in Figure 3, where the width of the channel is $d_{1}+d_{2}$. The fluid motion is considered to be induced by a sinusoidal wave train with a wave speed $c$ along the length of the channel walls. The walls equation is $Y=H_{1}$ and $Y=H_{2}$ maintained at the temperatures $T_{0}$ and $T_{1}$ and concentrations $C_{0}$ and $C_{1}$ respectively. The channel walls are represented by

$$
\begin{aligned}
& \mathrm{Y}=\mathrm{H}_{1}(\mathrm{X}, \mathrm{t})=\mathrm{d}_{1}+\mathrm{a}_{1} \operatorname{coc}\left(\frac{2 \pi}{\lambda^{*}}(X-\mathrm{ct})\right) \\
& \mathrm{Y}=\mathrm{H}_{2}(\mathrm{X}, \mathrm{t})=-\mathrm{d}_{2}-\mathrm{a}_{2} \cos \left(\frac{2 \pi}{\lambda^{*}}(X-c t)+\emptyset\right)
\end{aligned}
$$

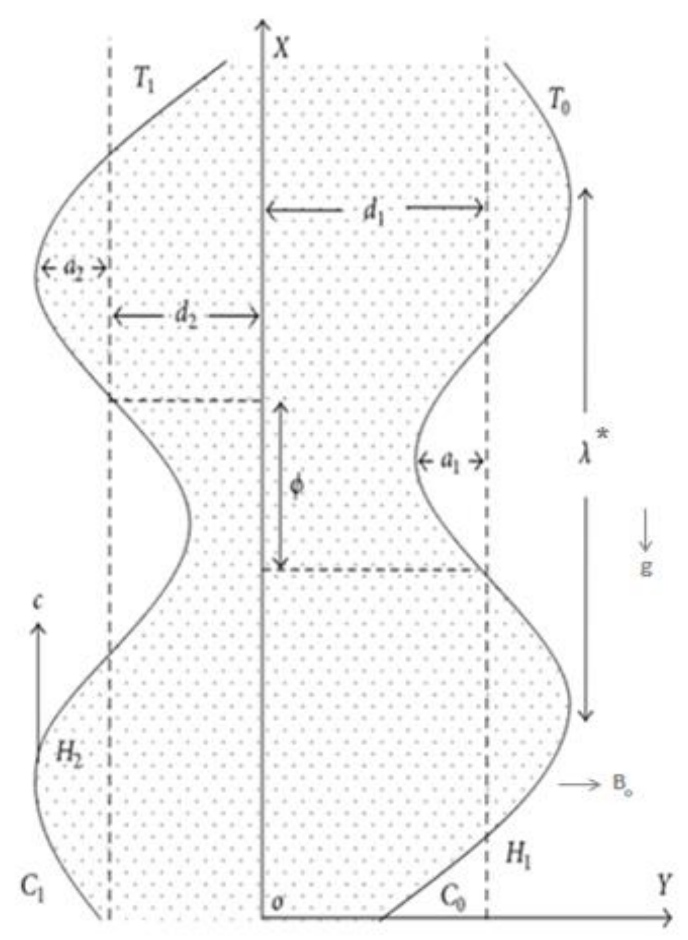

Fig. 3. Cartesian coordinates of 2Dimensional asymmetric channels

Where $a_{1}, a_{2}$ are the amplitudes of the waves, the phase difference $\emptyset$ varies in the range $0 \leq \emptyset \leq \pi, \emptyset$ $=0$ corresponds to the symmetric channel with waves out of phase and $\emptyset=\pi$ the waves are in the phase, and further $a_{1}, a_{2}, d_{1}, d_{2}$ and $\emptyset$ satisfy the condition

$$
a_{1}^{2}+b_{1}^{2}+2 a_{1} b_{1} \cos \emptyset \leq\left(d_{1}+d_{2}\right)^{2}
$$

The constitutive equation of the non-Newtonian Eyring-Powell model [18] is written as:

$$
\bar{\tau}=\mu \nabla V+\frac{1}{\beta} \sinh ^{-1}\left(\frac{1}{c_{1}} \nabla V\right)
$$

where $\bar{\tau}$ is extra stress tensor and $\mu$ is the dynamic viscosity $\beta$ and $c_{1}$ are the characteristics of the Eyring-Powell model [19]. Taking the second order approximation of the function $\sinh ^{-1}\left(\frac{1}{c_{1}} \nabla V\right)$ as 
$\sinh ^{-1}\left(\frac{1}{c_{1}} \nabla V\right)=\frac{1}{c_{1}} \nabla V-\frac{1}{6}\left(\frac{1}{c_{1}} \nabla V\right)^{3}, \quad\left|\frac{1}{c_{1}} \nabla V\right| \ll 1$

A uniform magnetic field with magnetic flux density $\vec{B}=\left(0, \mathrm{~B}_{0}, 0\right)$ is applied normal to axis of the channel. The expression for the current density $\vec{J}$ can be written as [20]:

$\vec{J}=\sigma^{*}(\vec{E}+\vec{V} \times \vec{B})$

where $\sigma^{*}$ is the electrical conductivity of the fluid it is measured in siemens unit, $\vec{V}$ is the velocity vector.

Assume that there is no applied polarization voltage so that the total electric field $\vec{E}$ can be neglected and the magnetic Reynolds number is very small so that the induced magnetic field can be neglected. The basic equations which describe this motion can be written as [20]

Continuity equation

$\frac{\partial U}{\partial X}+\frac{\partial V}{\partial Y}=0$

Momentum equations

$$
\begin{aligned}
\rho_{f}\left(\frac{\partial U}{\partial t}+U \frac{\partial U}{\partial X}+V \frac{\partial U}{\partial Y}\right)= & -\frac{\partial P}{\partial X}+\frac{\partial \tau_{X X}}{\partial X}+\frac{\partial \tau_{X Y}}{\partial Y}-\eta\left(\frac{\partial^{4} U}{\partial X^{4}}+\frac{\partial^{4} U}{\partial Y^{4}}\right)-\frac{\mu}{k_{o}} \mathrm{U}-\sigma^{*} B_{o}^{2} \mathrm{U}+ \\
& \rho_{f} \mathrm{~g} \beta_{t}\left(\mathrm{~T}-T_{o}\right)+\rho_{f} \mathrm{~g} \beta_{c}\left(\mathrm{C}-C_{o}\right) \\
\rho_{f}\left(\frac{\partial V}{\partial t}+U \frac{\partial V}{\partial X}+V \frac{\partial V}{\partial Y}\right)= & -\frac{\partial P}{\partial Y}+\frac{\partial \tau_{Y X}}{\partial X}+\frac{\partial \tau_{Y Y}}{\partial Y}-\eta\left(\frac{\partial^{4} V}{\partial X^{4}}+\frac{\partial^{4} V}{\partial Y^{4}}\right)-\frac{\mu}{k_{o}} \mathrm{~V}
\end{aligned}
$$

Heat equation

$$
\begin{aligned}
(\rho c)_{f}\left(\frac{\partial T}{\partial t}+U \frac{\partial T}{\partial X}+V \frac{\partial T}{\partial Y}\right)= & k\left(\frac{\partial^{2} T}{\partial X^{2}}+\frac{\partial^{2} T}{\partial Y^{2}}\right)+\frac{D_{m} k_{T}}{c_{S}}\left(\frac{\partial^{2} C}{\partial X^{2}}+\frac{\partial^{2} C}{\partial Y^{2}}\right)+\tau_{X X} \frac{\partial U}{\partial X}+\tau_{X Y} \frac{\partial U}{\partial Y}+ \\
& \tau_{Y X} \frac{\partial V}{\partial X}+\tau_{Y Y} \frac{\partial V}{\partial Y}+(\rho c)_{p}\left[\begin{array}{c}
D_{B}\left(\frac{\partial C}{\partial X} \frac{\partial T}{\partial X}+\frac{\partial C}{\partial Y} \frac{\partial T}{\partial Y}\right) \\
+\frac{D_{T}}{T_{o}}\left(\left(\frac{\partial T}{\partial X}\right)^{2}+\left(\frac{\partial T}{\partial Y}\right)^{2}\right)
\end{array}\right]-\frac{1}{(\rho c)_{p}} \frac{\partial q_{r}}{\partial Y}
\end{aligned}
$$

Concentration equation

$\left(\frac{\partial C}{\partial t}+U \frac{\partial C}{\partial X}+V \frac{\partial C}{\partial Y}\right)=D_{B}\left(\frac{\partial^{2} C}{\partial X^{2}}+\frac{\partial^{2} C}{\partial Y^{2}}\right)+\frac{D_{T}}{T_{o}}\left(\frac{\partial^{2} T}{\partial X^{2}}+\frac{\partial^{2} T}{\partial Y^{2}}\right)$

Where $\rho_{f}$ is the density of the fluid, $\mathrm{U}$ and $\mathrm{V}$ are the velocities in $\mathrm{X}$ and $\mathrm{Y}$ directions, $\mathrm{P}$ is the pressure in fixed frame, $k_{o}$ is the permeability of the porous medium, $\mathrm{T}$ is the temperature of the fluid, $\beta_{\mathrm{t}}$ is the thermal expansion coefficient, $\beta_{c}$ is the concentration expansion coefficient, $C$ is the nanoparticle concentration, $\mathrm{c}_{\mathrm{s}}$ concentration. The radiation term can be written as

$q_{r}=\frac{-4 \sigma_{s}}{3 k_{e}} \frac{\partial T^{4}}{\partial Y}$ 
where $\sigma_{s}$ represents the Stefan Boltzmann constant and $k_{e}$ is the mean absorption coefficient. Expanding the relation (12) by Tayler series, we get

$T^{4}=T_{\infty}^{4}+4 T_{\infty}^{3}\left(T-T_{\infty}\right)+6 T_{\infty}^{2}\left(T-T_{\infty}\right)^{2}+\cdots$

By neglecting higher-order terms in the above Eq. (13), one finds see $[22,25,26]$

$T^{4} \approx 4 T_{\infty}^{3} T-3 T_{\infty}^{4}$

Defining a wave frame $(x, y)$ with velocity $c$ from the fixed frame $(X, Y)$ with velocity components $u, v$ where the following transformation is considered

$\mathrm{x}=\mathrm{X}-\mathrm{ct}, \quad \mathrm{y}=\mathrm{Y}, \quad \mathrm{u}=\mathrm{U}-\mathrm{c}, \quad \mathrm{v}=\mathrm{V} \quad$ and $\quad \mathrm{p}(\mathrm{x})=\mathrm{P}(\mathrm{X}, \mathrm{t})$

Introducing the following non-dimensional variables

$$
\left.\begin{array}{c}
\bar{x}=\frac{x}{\lambda^{*}}, \quad \bar{y}=\frac{y}{d_{1}}, \quad \bar{u}=\frac{u}{c}, \bar{v}=\frac{v}{c \delta}, \delta=\frac{d_{1}}{\lambda^{*}}, \bar{p}=\frac{d_{1}^{2} p}{\mu c \lambda^{*}}, \bar{t}=\frac{c t}{\lambda^{*}}, \\
h_{1}=\frac{H_{1}}{d_{1}}, h_{2}=\frac{H_{2}}{d_{2}}, d=\frac{d_{2}}{d_{1}}, a=\frac{a_{1}}{d_{1}}, b=\frac{a_{2}}{d_{1}}, R e=\frac{\rho_{f} c d_{1}}{\mu}, \quad \theta=\frac{T-T_{o}}{T_{1}-T_{0}}, \\
\sigma=\frac{c-C_{o}}{c_{1}-C_{o}}, S^{2}=\frac{\mu_{1} d_{1}^{2}}{\eta}, G_{r}=\frac{\rho_{f} g \beta_{t} d_{1}^{2}\left(T_{1}-T_{o}\right)}{c \mu}, \gamma=\frac{k_{1} d_{1}^{2}}{\mu} \\
G_{c}=\frac{\rho_{f} g \beta_{c} d_{1}^{2}\left(C_{1}-C_{o}\right)}{c \mu}, M^{2}=\frac{\sigma^{*} B_{o}^{2} d_{1}^{2}}{\mu}, p_{r=} \frac{C_{p} \mu}{k}, k=\frac{k_{o}}{d_{1}^{2}} \\
, \bar{\tau}_{x x}=\frac{\lambda^{*}}{\mu c} \tau_{x x}, \bar{\tau}_{x y}=\frac{d_{1}}{\mu c} \tau_{x y}, \bar{\tau}_{y y}=\frac{d_{1}}{\mu c} \tau_{y y}, \\
, E_{c}=\frac{c^{2}}{C_{p}\left(T_{1}-T_{o}\right)}, R_{n}=\frac{k_{e} k}{4 \sigma_{s} T_{\infty}^{3}}
\end{array}\right\}
$$

Using these dimensionless variables in Eq. (16) that illustrated above the basic Eq. (7)-(11) after dropping the bars, can be written as:

$$
\begin{aligned}
& \frac{\partial u}{\partial x}+\frac{\partial v}{\partial y}=0 \\
& \operatorname{Re\delta }\left(u \frac{\partial u}{\partial x}+v \frac{\partial u}{\partial y}\right)=-\frac{\partial p}{\partial x}+\frac{d_{1}^{2}}{\mu c}\left(\frac{\partial \tau_{x x}}{\partial x}+\frac{\partial \tau_{x y}}{\partial y}\right)-\frac{1}{s^{2}}\left(\delta^{4} \frac{\partial^{4} u}{\partial x^{4}}+\frac{\partial^{4} u}{\partial y^{4}}\right)- \\
& \left(\frac{1}{k}+M^{2}\right) u+G_{r} \theta+G_{c} \sigma
\end{aligned}
$$

$R e \delta^{3}\left(u \frac{\partial v}{\partial x}+v \frac{\partial v}{\partial y}\right)=-\frac{\partial p}{\partial y}+\frac{d_{1}^{2} \delta}{\mu c}\left(\frac{\partial \tau_{y x}}{\partial x}+\frac{\partial \tau_{y y}}{\partial y}\right)-\frac{\delta^{2}}{s^{2}}\left(\delta^{4} \frac{\partial^{4} v}{\partial x^{4}}+\frac{\partial^{4} v}{\partial y^{4}}\right)-\frac{\delta^{2}}{k} v$

$R e P_{r} \delta\left(u \frac{\partial \theta}{\partial x}+v \frac{\partial \theta}{\partial y}\right)=\left(\delta^{2} \frac{\partial^{2} \theta}{\partial x^{2}}+\frac{\partial^{2} \theta}{\partial y^{2}}\right)+D_{u}\left(\delta^{2} \frac{\partial^{2} \sigma}{\partial x^{2}}+\frac{\partial^{2} \sigma}{\partial y^{2}}\right)+\frac{d_{1}^{2}}{k\left(T_{1}-T_{o}\right)}\left(\tau_{x x} \frac{\partial u}{\partial x}+\right.$

$$
\left.\tau_{x y} \frac{\partial u}{\partial y}+\tau_{y x} \frac{\partial v}{\partial x}+\tau_{y y} \frac{\partial v}{\partial y}\right)+\left[\frac{N_{b}}{P_{r}}\left(\frac{\partial \sigma}{\partial y} \frac{\partial \theta}{\partial y}\right)+\frac{N_{t}}{P_{r}}\left(\frac{\partial \theta}{\partial y}\right)^{2}\right]-\frac{4}{3 R_{n}} \frac{\partial^{2} \theta}{\partial y^{2}}
$$

$\operatorname{Re}_{c} S_{c}\left(u \frac{\partial \sigma}{\partial x}+v \frac{\partial \sigma}{\partial y}\right)=\left(\delta^{2} \frac{\partial^{2} \sigma}{\partial x^{2}}+\frac{\partial^{2} \sigma}{\partial y^{2}}\right)+\frac{N_{t}}{N_{b}}\left(\delta^{2} \frac{\partial^{2} \theta}{\partial x^{2}}+\frac{\partial^{2} \theta}{\partial y^{2}}\right)$ 
where $\mathrm{p}$ is the dimensionless pressure, $\mathrm{k}$ is the porosity parameter, $\mathrm{S}$ is the couple stress parameter, $G r$ is the local temperature Grashof number, $G_{c}$ is the local mass Grashof number, $P_{r}$ is the Prandt number, $\mathrm{S}_{\mathrm{c}}$ is the Schmidt number, $\mathrm{k}$ is the thermal conductivity.

Using the approximations of the long wavelength $(\delta<1$ because the wavelength is inversely proportional to the wave number, the $\delta=\frac{d_{1}}{\lambda^{*}}$ ) and small Reynolds number ( $\operatorname{Re} \rightarrow 0$ ) then Eq. (18)(21) can be rewritten as

$$
\begin{aligned}
& -\frac{\partial p}{\partial x}+(1+m)\left(\frac{\partial^{2} u}{\partial y^{2}}\right)-m \lambda\left(\frac{\partial u}{\partial y}\right)^{2} \frac{\partial^{2} u}{\partial y^{2}}-\frac{1}{s^{2}}\left(\frac{\partial^{4} u}{\partial y^{4}}\right)-\left(\frac{1}{k}+M^{2}\right) u+G_{r} \theta+G_{c} \sigma=0 \\
& \frac{\partial P}{\partial y}=0 \\
& \left(1+\frac{4}{3 R_{n}}\right)\left(\frac{\partial^{2} \theta}{\partial y^{2}}\right)+P_{r} D_{u}\left(\frac{\partial^{2} \sigma}{\partial y^{2}}\right)+N_{b}\left(\frac{\partial \sigma}{\partial y} \frac{\partial \theta}{\partial y}\right)+N_{t}\left(\frac{\partial \theta}{\partial y}\right)^{2}+P_{r} E_{c}\left[(1+m)\left(\frac{\partial u}{\partial y}\right)^{2}-\right. \\
& \left.\frac{1}{3} m \lambda\left(\frac{\partial u}{\partial y}\right)^{4}\right]=0 \\
& \left(\frac{\partial^{2} \sigma}{\partial y^{2}}\right)+\frac{N_{t}}{N_{b}}\left(\frac{\partial^{2} \theta}{\partial y^{2}}\right)=0
\end{aligned}
$$

where $m=\frac{1}{\rho \beta v c_{1}}$ is the fluid material parameter and $\lambda=\frac{\rho c^{2}}{2 c_{1}^{2} d^{2}}$ is the local non-Newtonian parameter based on velocity of plate $c$.

\section{Model Solution}

Differentiation Eq. (22) with respect to $y$, and Eq. (23) with respect to $x$, then cancel the pressure from Eq. (22) and Eq. (23) and integrate respect to y we have

\begin{tabular}{|c|c|c|c|c|c|c|}
\hline $\mathrm{u}=-1$ & at & $y=h_{1}$ & and & $y=h_{2}$ & & \\
\hline$u^{\prime \prime}=0$ & at & $y=h_{1}$ & and & $y=h_{2}$ & & \\
\hline$\theta=0$ & at & $y=h_{1}$ & and & $\theta=1$ & at & $y=h_{2}$ \\
\hline$\sigma=0$ & at & $y=h_{1}$ & and & $\sigma=1$ & at & $y=h_{2}$ \\
\hline
\end{tabular}

$\frac{1}{s^{2}}\left(\frac{\partial^{4} u}{\partial y^{4}}\right)-(1+m)\left(\frac{\partial^{2} u}{\partial y^{2}}\right)+m \lambda\left(\frac{\partial u}{\partial y}\right)^{2} \frac{\partial^{2} u}{\partial y^{2}}+\left(\frac{1}{k}+M^{2}\right) u-G_{r} \theta-G_{C} \sigma=R$

where $\mathrm{R}$ is the constant of integrate. The appositely corresponding boundary conditions in dimensionless form are

where $h_{1}=1+a \cos (2 \pi x), h_{2}=-d-b \cos (2 \pi x+\emptyset)$.

The system of Eq. (24)-(26) subjected to the conditions from Eq. (27)-(30) are solved numerically by using A Rung-Kutta-Merson method. Then the velocity, temperature and concentration of the fluid are obtained numerically. The effects of physical parameters of the problem on these distributions are discussed numerically and illustrated graphically through a set of figures. 


\section{Results and Discussion}

The effects of the physical parameters of the current work on the peristaltic motion of EyringPowell nanofluid with heat and mass transfer in the presence of couple stresses with magnetic field through asymmetric channel are discussed. The velocity, temperature and concentration of the fluid are obtained numerically by using Rung-Kutta-Merson method. The effects of Hartmann number $\mathrm{M}$, Brownain motion parameter $N_{b}$, thermophoresis parameter $N_{t}$, porosity parameter $k$, fluid parameter $m$, local non-Newtonian parameter $\lambda$ and Couple stress parameter $S$ on the velocity $u$ are show through Figure 4 to Figure 10. Figure 4 is plotted to see the variation of velocity for different values of $\mathrm{M}$. It is observed that the velocity profiles are parabolic in nature. It is observed from this figure that the velocity profile decreases when $M$ increasing. This seems realistic, because the magnetic field acts in the transverse direction to the flow and magnetic force resists the flow. The same behavior can be seen with increasing $N_{t}, \lambda$ and $k$ opposite behavior has seen for $\mathrm{S}$ see Figure 5 to Figure 8 . The velocity increasing with increasing $N_{b}$ see Figure 9 . The same behavior can be seen with increasing $m$. Figure 10 is drawn to study the effect of $m$ for the velocity distribution. It is noticed that when $m$ increasing the fluid becomes less viscous hence the velocity increasing.

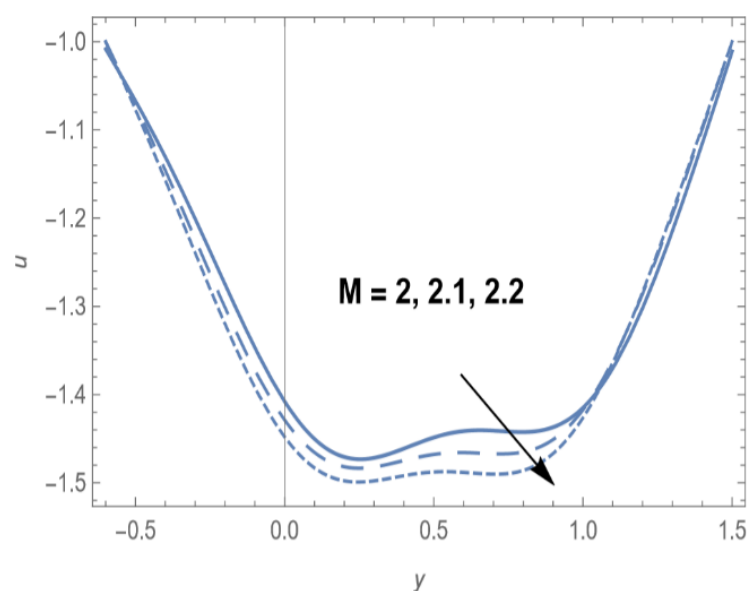

Fig. 4. The velocity profile $\mathrm{u}$ is plotted against $\mathrm{y}$ for several values of $M$ for $m=3, \lambda=2.4, R_{n}=$ $0.5, N_{b}=0.1, N_{t}=0.5, p_{r}=0.01, E_{c}=0.1, D_{u}=$ $0.5, s=2, R=2, k=2, g_{r}=0.7, g_{c}=0.9$

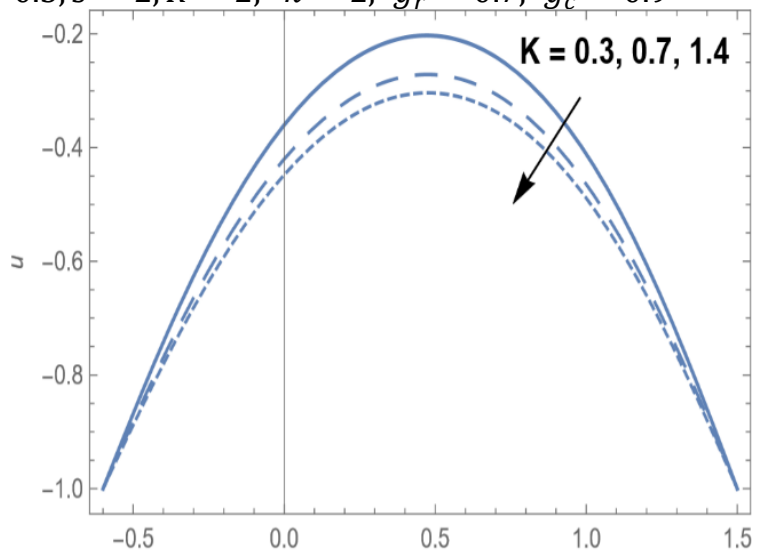

Fig. 6. The velocity profile $\mathrm{u}$ is plotted against $\mathrm{y}$ for several values of $k$ for $m=1, \lambda=1.4, R_{n}=$ $0.5, N_{b}=0.1, \mathrm{M}=2, p_{r}=0.01, E_{c}=0.1, D_{u}=$ $0.5, s=2, R=0.05, N_{t}=0.5, g_{r}=0.7, g_{c}=$ 0.9

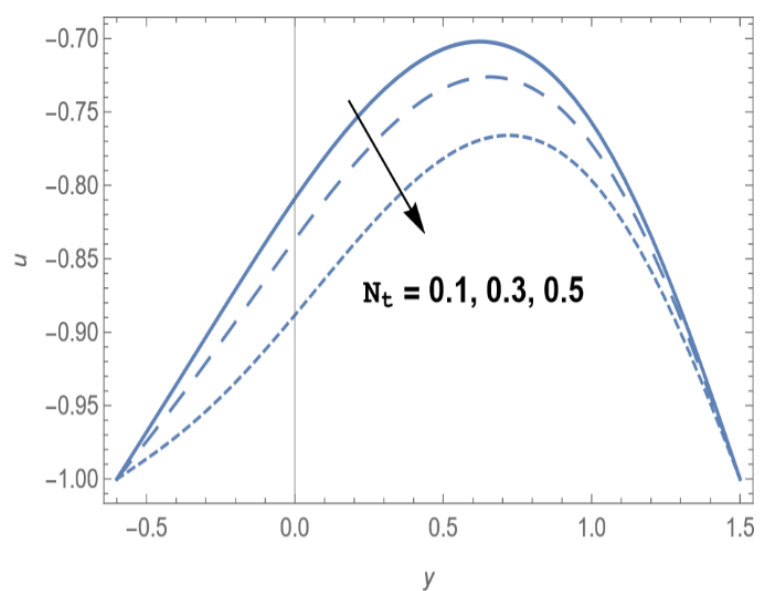

Fig. 5. The velocity profile $u$ is plotted against $y$ for several values of $N_{t}$ for $m=3, \lambda=2, R_{n}=$ $1, N_{b}=0.7, \mathrm{M}=0.1, p_{r}=1, E_{c}=0.8, D_{u}=$ $0.5, s=2, R=0.05, k=2, g_{r}=0.7, g_{c}=0.9$

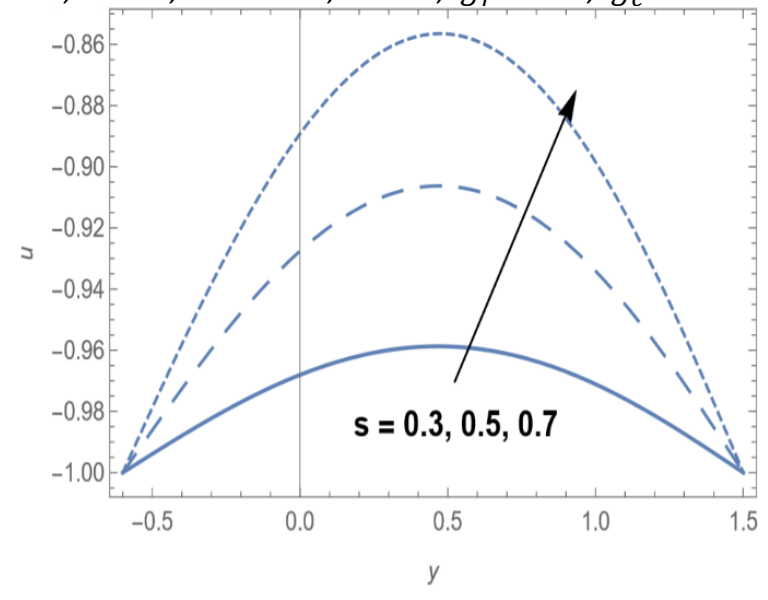

Fig. 7. The velocity profile $u$ is plotted against $y$ for several values of $s$ for $m=3, \lambda=0.2, R_{n}=$ $0.5, N_{b}=0.1, \mathrm{M}=0.1, p_{r}=0.01, E_{c}=$ $0.1, D_{u}=0.5, k=2, R=1, N_{t}=0.5, g_{r}=$ 0.7, $g_{c}=0.9$ 


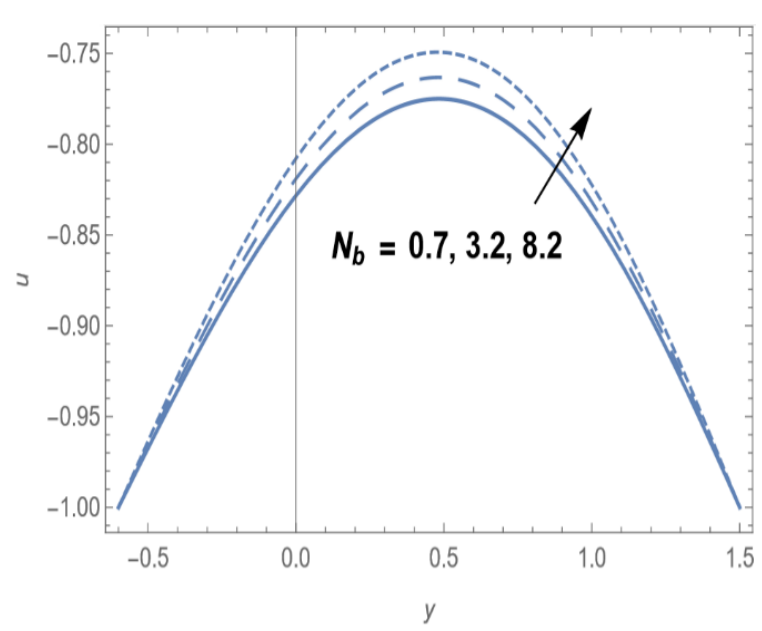

Fig. 8. The velocity profile $u$ is plotted against $y$ for several values of $N_{b}$ for $m=0.1, \lambda=2$, $R_{n}=0.5, \mathrm{~s}=1, \mathrm{M}=0.1, p_{r}=1, E_{c}=$ $0.1, D_{u}=0.5, k=2, R=0.05, N_{t}=0.5$, $g_{r}=0.7, g_{c}=0.9$

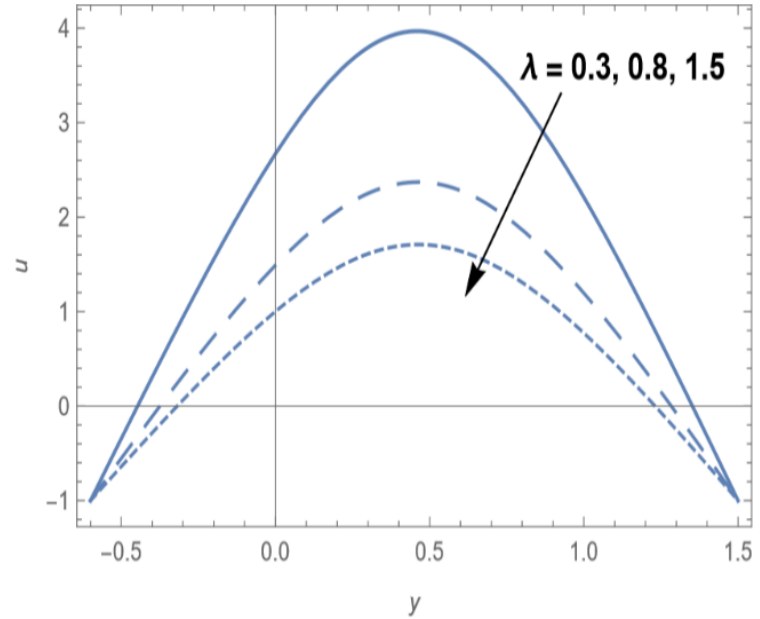

Fig. 9. The velocity profile $u$ is plotted against $y$ for several values of $\lambda$ for $m=1, N_{b}=0.1$, $R_{n}=0.5, \mathrm{~s}=2, \mathrm{M}=0.1, p_{r}=0.01, E_{c}=$ $0.1, D_{u}=0.5, k=0.4, R=0.05, N_{t}=0.5$, $g_{r}=0.7, g_{c}=0.9$

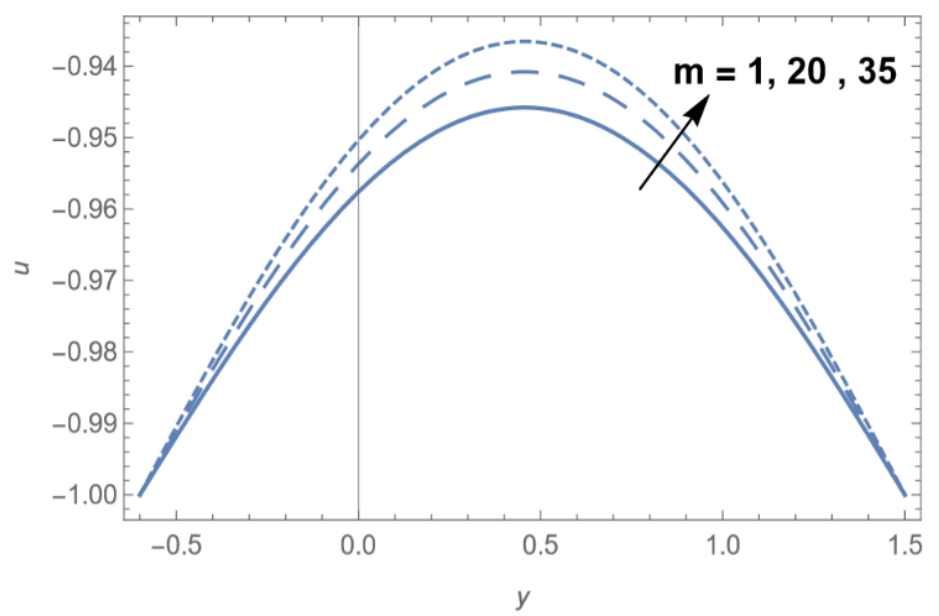

Fig. 10. The velocity profile $u$ is plotted against $y$ for several values of $m$ for $\lambda=1.4, N_{b}=0.1, R_{n}=0.5, \mathrm{~s}=0.2$, $\mathrm{M}=0.1, p_{r}=0.01, E_{c}=0.1, D_{u}=0.5, k=0.2, R=$ $0.05, N_{t}=0.5, g_{r}=0.7, g_{c}=0.9$

The variation of temperature profile for different values of Brownain motion parameter $N_{b}$, local non-Newtonian parameter $\lambda$, Couple stress parameter $S$ and Radiation parameter $R_{n}$, are plotted in Figure 11 and Figure 14. It is clear that the temperature profile increasing with increasing both of $N_{b}$ and $\mathrm{S}$, opposite behavior has seen for $\lambda$ and $R_{n}$ because the temperature is inversely proportional to the Radiation parameter, so when Radiation parameter increasing the temperature profile decreases. 


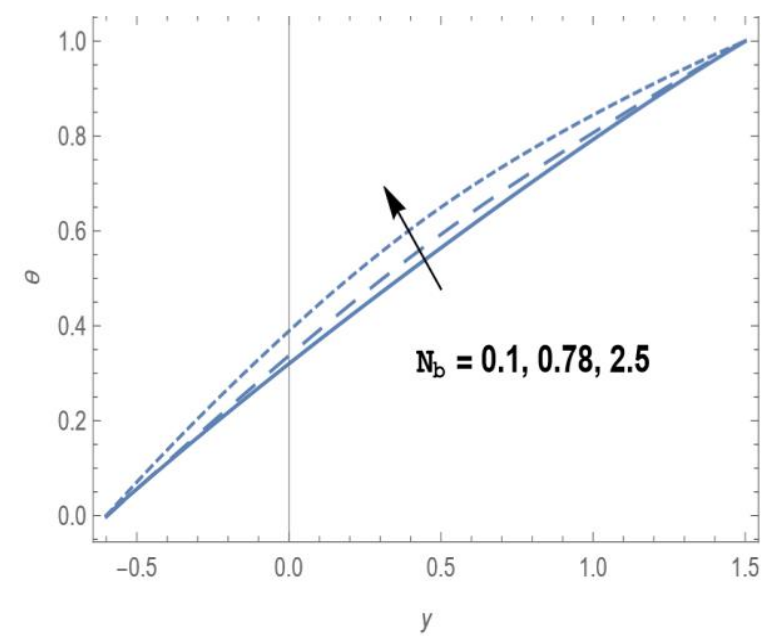

Fig. 11. The variation of temperature profile $\theta$ is plotted against $\mathrm{y}$ for several values of $N_{b}$ for $m=3, \lambda=2, R_{n}=0.5, \mathrm{~s}=2, \mathrm{M}=0.1$, $p_{r}=0.01, E_{c}=0.1, D_{u}=0.5, k=2, R=$ $0.05, N_{t}=0.5, g_{r}=0.7, g_{c}=0.9$

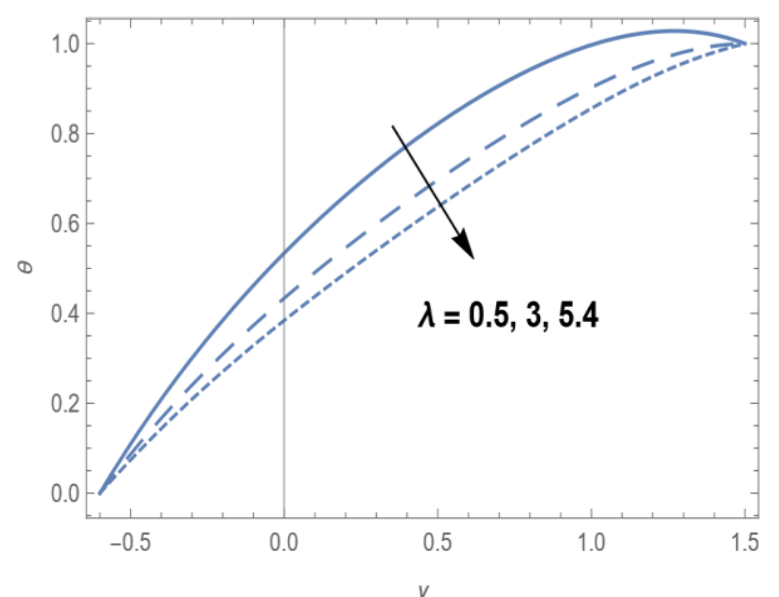

Fig. 13. The variation of temperature profile $\theta$ is plotted against $\mathrm{y}$ for several values of $\lambda$ for $m=$ 2, $N_{b}=0.1, R_{n}=2, \mathrm{~s}=2, \mathrm{M}=1.5, p_{r}=3$, $E_{c}=0.1, D_{u}=0.05, k=0.04, R=0.4$, $N_{t}=0.5, g_{r}=0.7, g_{c}=0.9$

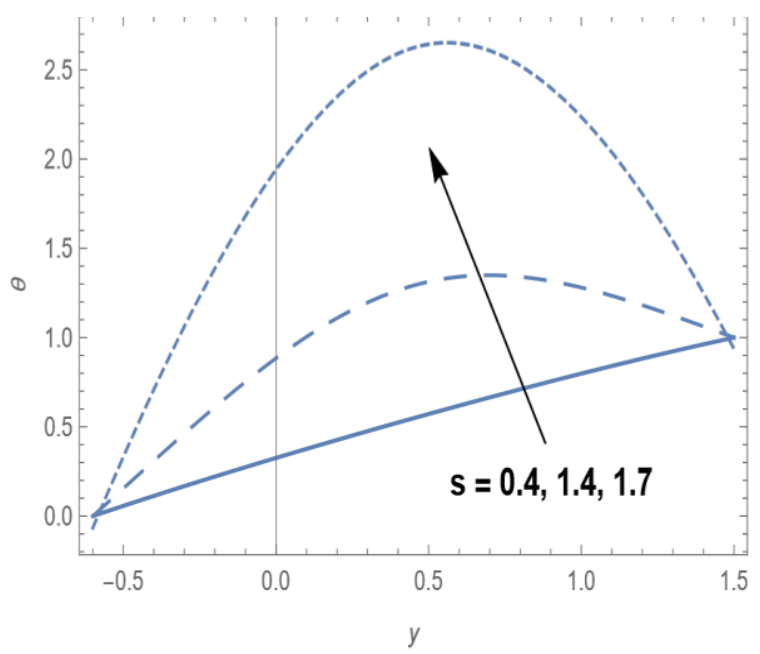

Fig. 12. The variation of temperature profile $\theta$ is plotted against y for several values of $s$ for $m=$ $4, \lambda=0.2, R_{n}=0.5, N_{b}=0.1, \mathrm{M}=0.1$, $p_{r}=0.01, E_{c}=0.1, D_{u}=0.5, k=0.2, R=$ 5, $N_{t}=0.5, g_{r}=0.7, g_{c}=0.9$

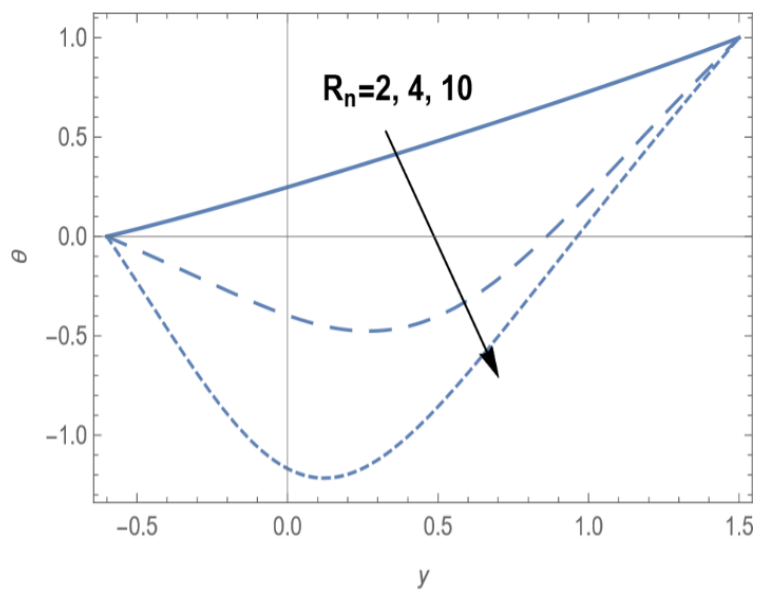

Fig. 14. The variation of temperature profile $\theta$ is plotted against $\mathrm{y}$ for several values of $R_{n}$ for $m=1, \lambda=0.1, \quad N_{b}=1, \mathrm{~s}=2, \mathrm{M}=1.5$, $p_{r}=3, E_{c}=0.1, D_{u}=0.05, k=0.4, R=$ $0.05, N_{t}=0.5, g_{r}=0.7, g_{c}=0.9$

The variation of Concentration profile with different values of the thermophoresis parameter $N_{t}$ and radiation parameter $R_{n}$ plotted in Figure 15 and Figure 16. It is clear that the Concentration profile decreasing with increasing of both $N_{t}$ and $R_{n}$. 


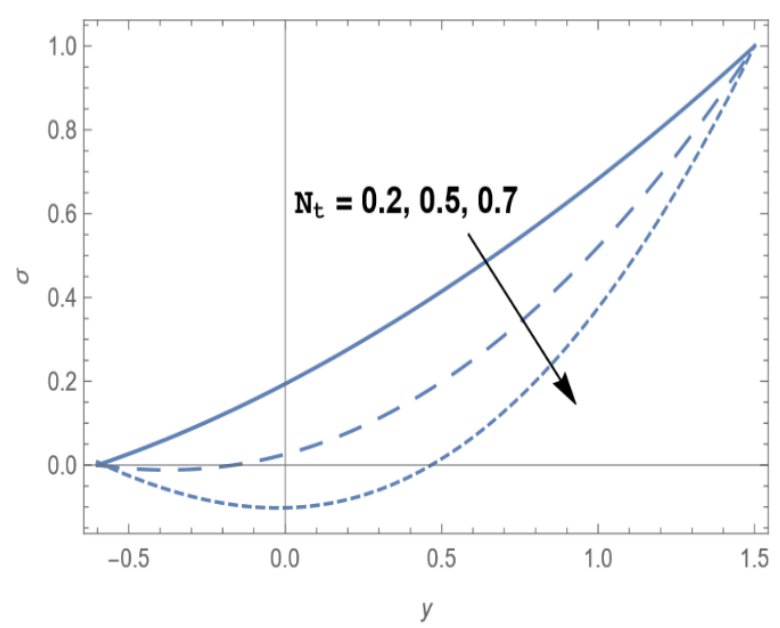

Fig. 15. The concentration profile $\sigma$ is plotted against $y$ for several values of $N_{t}$ for $m=3, \lambda=$ 2, $R_{n}=1, N_{b}=1, \mathrm{M}=0.1, p_{r}=1, E_{c}=$ $0.8, D_{u}=0.5, s=2, R=0.05, k=2, g_{r}=$ $0.7, g_{c}=0.9$

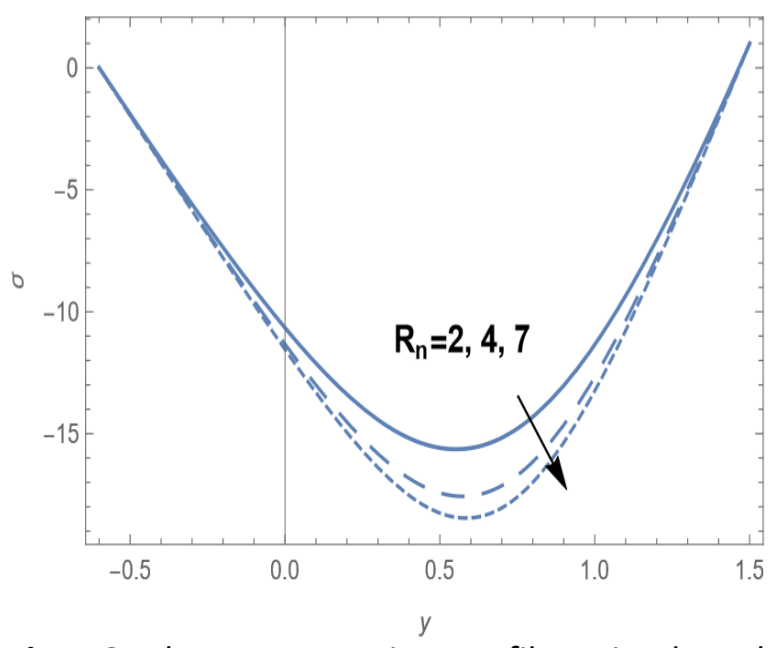

Fig. 16. The concentration profile $\sigma$ is plotted against $\mathrm{y}$ for several values of $R_{n}$ for $m=1, \lambda=$ 2, $N_{b}=0.1, \mathrm{~s}=0.1, \mathrm{M}=1.5, p_{r}=0.01$, $E_{c}=0.1, D_{u}=0.5, k=0.4, R=0.05, N_{t}=$ 2.5, $g_{r}=0.7, g_{c}=0.9$

\section{Conclusions}

Current study is to analyse the combined effects of the Hartmann number, Brownain motion parameter, thermophoresis parameter, porosity parameter, radiation parameter, fluid parameter, local non-Newtonian parameter and Couple stress parameter on the obtained solutions of the peristaltic motion of non-Newtonian nano fluid through porous media inside asymmetric channel, The system of equations are solved numerically by using A Rung-Kutta-Merson method under the long wave length and low Reynolds number approximations. The velocity, temperature and concentration distributed of the fluid are obtained as a function of the physical parameters of the problem. The effects of physical parameters of the problem on these distributions are discussed numerically and illustrated graphically through a set of figures. The very important results are summarized as

i. The velocity increasing with increasing the Brownain motion parameter, Couple stress parameter and fluid material parameter. It is noticed that when fluid parameter increasing the fluid becomes less viscous hence the velocity increasing.

ii. The velocity decreasing with increasing the local non-Newtonian parameter, thermophoresis parameter, porosity parameter and Hartmann number, because the magnetic field acts in the transverse direction to the flow and magnetic force resists the flow.

iii. The temperature increases with increasing both of the Couple stress parameter and Brownain motion parameter.

iv. The temperature decreasing with increasing in local non-Newtonian parameter and radiation parameter, because the temperature is inversely proportional to the Radiation parameter, so when radiation parameter increasing the temperature profile decreases $\left(R_{n}=\frac{k_{e} k}{4 \sigma_{s} T_{\infty}^{3}}\right)$.

v. The concentration decreases with increasing both of radiation parameter and thermophoresis parameter. 


\section{References}

[1] Kothandapani, M., and J. Prakash. "Effects of thermal radiation parameter and magnetic field on the peristaltic motion of Williamson nanofluids in a tapered asymmetric channel." International Journal of Heat and Mass Transfer 81 (2015): 234-245.

https://doi.org/10.1016/j.ijheatmasstransfer.2014.09.062

[2] Kuznetsov, A. V., and D. A. Nield. "Natural convective boundary-layer flow of a nanofluid past a vertical plate." International Journal of Thermal Sciences 49, no. 2 (2010): 243-247. https://doi.org/10.1016/j.ijthermalsci.2009.07.015

[3] Akbar, Noreen Sher, and S. Nadeem. "Endoscopic effects on peristaltic flow of a nanofluid." Communications in Theoretical Physics 56, no. 4 (2011): 761.

https://doi.org/10.1088/0253-6102/56/4/28

[4] Gireesha, B. J., B. Mahanthesh, and Rama Subba Reddy Gorla. "Suspended particle effect on nanofluid boundary layer flow past a stretching surface." Journal of nanofluids 3, no. 3 (2014): 1-11.

https://doi.org/10.1166/jon.2014.1101

[5] Akbar, Noreen Sher, S. Nadeem, T. Hayat, and Awatif A. Hendi. "Peristaltic flow of a nanofluid in a non-uniform tube." Heat and Mass Transfer 48, no. 3 (2012): 451-459.

https://doi.org/10.1007/s00231-011-0892-7

[6] Nadeem, Sohail, Arshad Riaz, Rahmat Ellahi, and Noreen Sher Akbar. "Effects of heat and mass transfer on peristaltic flow of a nanofluid between eccentric cylinders." Applied Nanoscience 4, no. 1 (2010): 26-40.

[7] Hina, S., M. Mustafa, T. Hayat, and A. Alsaedi. "Peristaltic flow of couple-stress fluid with heat and mass transfer: An application in biomedicine." Journal of Mechanics in Medicine and Biology 15, no. 04 (2015): 1-17. https://doi.org/10.1142/S0219519415500426

[8] Akbar, Noreen Sher. "Peristaltic flow of a tangent hyperbolic fluid with convective boundary condition." The European Physical Journal Plus 129, no. 10 (2014): 214.

https://doi.org/10.1140/epjp/i2014-14214-0

[9] Nadeem, S., Sadaf Ashiq, Noreen Sher Akbar, and Changhoon Lee. "Peristaltic flow of hyperbolic tangent fluid in a diverging tube with heat and mass transfer." Journal of Energy Engineering 139, no. 2 (2013): 124-135. https://doi.org/10.1061/(ASCE)EY.1943-7897.0000094

[10] Eldabe, Nabil T., and Mohamed Y. Abou-zeid. "Magnetohydrodynamic peristaltic flow with heat and mass transfer of micropolar biviscosity fluid through a porous medium between two co-axial tubes." Arabian Journal for Science and Engineering 39, no. 6 (2014): 5045-5062.

https://doi.org/10.1007/s13369-014-1039-1

[11] Eldabe, Nabil, and Mohamed Abou-Zeid. "Radially varying magnetic field effect on peristaltic motion with heat and mass transfer of a non-Newtonian fluid between two co-axial tubes." Thermal Science 22, no. 6 Part A (2018): 24492458. https://doi.org/10.2298/TSCI160409292E

[12] Eldabe, Nabil TM, Sallam N. Sallam, and Mohamed Y. Abou-zeid. "Numerical study of viscous dissipation effect on free convection heat and mass transfer of MHD non-Newtonian fluid flow through a porous medium." Journal of the Egyptian mathematical society 20, no. 2 (2012): 139-151. https://doi.org/10.1016/j.joems.2012.08.013

[13] El-Dabe, N. S. Sallam, Mona AA Mohamed, Y. A. Mohamed and Asmaa A. H. "Effects of Chemical Reaction on Peristaltic Motion of MHD Biviscosity Fluid with Heat and Mass Transfer through Porous Medium in an Inclined Asymmetric Channel." Int. J. Appl. Math. Phys 3 (2011) : 137-146.

[14] Abou-zeid, Mohamed. "Magnetohydrodynamic boundary layer heat transfer to a stretching sheet including viscous dissipation and internal heat generation in a porous medium." Journal of porous Media 14, no. 11 (2011): 10071018.

https://doi.org/10.1615/JPorMedia.v14.i11.50

[15] Eldabe, N. T., M. A. Elogail, S. M. Elshaboury, and Alfaisal A. Hasan. "Hall effects on the peristaltic transport of Williamson fluid through a porous medium with heat and mass transfer." Applied Mathematical Modelling 40, no. 1 (2016): 315-328. https://doi.org/10.1016/i.apm.2015.04.043

[16] Ramesh, K., and M. Devakar. "Magnetohydrodynamic peristaltic transport of couple stress fluid through porous medium in an inclined asymmetric channel with heat transfer." Journal of Magnetism and Magnetic Materials 394 (2015): 335-348. https://doi.org/10.1016/j.jmmm.2015.06.052 
[17] Hina, S., M. Mustafa, T. Hayat, and A. Alsaedi. "Peristaltic transport of Powell-Eyring fluid in a curved channel with heat/mass transfer and wall properties." International Journal of Heat and Mass Transfer 101 (2016): 156-165. https://doi.org/10.1016/i.ijheatmasstransfer.2016.05.034

[18] Powell, Richard E., and Henry Eyring. "Mechanisms for the relaxation theory of viscosity." Nature 154, no. 3909 (1944): 427-428. https://doi.org/10.1038/154427a0

[19] Zaman, Haider, Murad Ali Shah, and Muhammad Ibrahim. "Unsteady incompressible Couette flow problem for the Eyring-Powell model with porous walls." American Journal of Computational Mathematics 3 (2013) : 313-325. https://doi.org/10.4236/ajcm.2013.34041

[20] Eldabe, Nabil TM, Osama M. Abo-Seida, Adel AS Abo-Seliem, A. A. ElShekhipy, and Nada Hegazy. "Peristaltic transport of magnetohydrodynamic carreau nanofluid with heat and mass transfer inside asymmetric channel." American Journal of Computational Mathematics 7, no. 01 (2017): 1-20. https://doi.org/10.4236/ajcm.2017.71001

[21] Govindarajan, A., E. P. Siva, and M. Vidhya. "Combined effect of heat and mass transfer on MHD Peristaltic transport of a couple stress fluid in a inclined asymmetric channel through a porous medium." International Journal of Pure and Applied Mathematics 105, no. 4 (2015): 685-707.

https://doi.org/10.12732/ijpam.v105i4.9

[22] Eldabe, Nabil TM, Ahmed Y. Ghaly, Sallam N. Sallam, Khaled Elagamy, and Yasmeen M. Younis. "Hall effect on Peristaltic flow of third order fluid in a porous medium with heat and mass transfer." Journal of Applied Mathematics and Physics 3, no. 09 (2015): 1138-1150. https://doi.org/10.4236/jamp.2015.39141

[23] Yaari, Z., A. Zinger, E. Goldman, A. Kajal, R. Tshuva, E. Barak, N. Dahan et al. "Theranostic barcoded nanoparticles for personalized cancer medicine." Nature communications 7 (2016): 13325. https://doi.org/10.1038/ncomms13325

[24] Liu, Lejie, Shahrzad Towfighian, and Amine Hila. "A review of locomotion systems for capsule endoscopy." IEEE reviews in biomedical engineering 8 (2015): 138-151. https://doi.org/10.1109/RBME.2015.2451031

[25] Alharbi, Sayer Obaid, Abdullah Dawar, Zahir Shah, Waris Khan, Muhammad Idrees, Saeed Islam, and I. Khan. "Entropy generation in MHD eyring-powell fluid flow over an unsteady oscillatory porous stretching surface under the impact of thermal radiation and heat source/sink." Applied Sciences 8, no. 12 (2018): 2588.

[26] Khan, S. U., N. Ali, and Z. Abbas. "Hydromagnetic Flow and Heat Transfer of Eyring-Powell Fluid over an Oscillatory Stretching Sheet with Thermal Radiation." Applications \& Applied Mathematics 10, no. 2 (2015): 893-908. 